\title{
Consumo de sustancias y conductas de riesgo en consumidores de pasta base de cocaína y clorhidrato de cocaína no consultantes a servicios de rehabilitación
}

\author{
Rodrigo Santis B ${ }^{1}$, Carmen G loria Hidalgo $C^{2 a}$, Viviana Hayden $C^{1 a}$, \\ Enzo Anselmo $\mathrm{M}^{\mathrm{la}}$, Jorge Rodríguez $\mathrm{T}^{3 \mathrm{~b}}$, \\ Fernando Cartajena de la $M^{1 c}$, Jorge Dreyse $D^{1 c}$, Rafael Torres $B^{1}$. \\ Substance use and risk behaviors of \\ out-of-treatment cocaine base paste \\ and cocaine hydrochloride users
}

Background: In Chile, cocaine base paste (CBP) is the illegal substance that produces the highest rate of addiction. Nonetheless, a marginal number of users receive treatment each year. Aim: To compare the consumption patterns and risk behavior of CBP and cocaine hydrochloride (CH) users who do not attend rehabilitation services. Material and Methods: In a prospective research design, through a study methodology called Privileged Access Interview of hidden populations, 28 surveyors recruited $231 \mathrm{CBP}$ users (group 1) and $236 \mathrm{CH}$ users (group 2). The Risk Behavior Questionnaire was applied in four communities of Metropolitan Santiago, that have the highest prevalence of PBC and $\mathrm{CH}$ use. Results: CBP users showed higher schools drop-out and unemployment rates. Subjects of both groups were predominantly polysubstance and polyaddicted users. The severity of addiction to CBP of group 1 was significantly higher than the severity of addiction to $\mathrm{CH}$ of group 2 ( 5.5 versus 5.1: $\mathrm{p}<0.001$ ). CBP users showed significantly higher rates of sexual risk behaviors, antisocial behavior, self infliction of injuries, suicide attempt and child neglect. Conclusions: A higher vulnerability was shown for users of $\mathrm{CBP}$ than those of $\mathrm{CH}$. Attention is drawn to the need for developing community interventions in order to alter substance abuse and the risk behavior of these vulnerable groups (Rev Méd Chile 2007; 135: 45-53).

(Key words: Behavior; Cocaine; Cocaine related disorders)

\footnotetext{
Recibido el 13 de marzo, 2006. Aceptado el 27 de junio, 2006.

Trabajo financiado por proyecto FONDECYT (\#1030431). La organización que financió el proyecto no tuvo influencia en el diseño, ni en la ejecución del estudio, como tampoco en la preparación de este manuscrito.

${ }^{1}$ Departamento de Psiquiatría, Facultad de Medicina, Pontificia Universidad Católica de Chile. ${ }^{2}$ Escuela de Psicología, Facultad de Ciencias Sociales, Pontificia Universidad Católica de Chile. ${ }^{3}$ Escuela de Salud Pública, Facultad de Medicina, Universidad de Chile.

aPsicólogo

bEstadístico

cAlumno, Escuela de Medicina, Pontificia Universidad Católica de Chile.
}

Correspondencia a: Dr. Rodrigo Santis B. Camino El Alba 12351, Las Condes. CP 762-0002. Santiago, Chile. Casilla: 114-D. Fono: 7548872. Fax: 7548878. E mail: rsantis@med.puc.cl 
$\mathrm{E}^{\mathrm{n}}$ Chile, la cocaína es la segunda droga ilegal de mayor consumo, alcanzando $1,9 \%$ de prevalencia anual de consumo ${ }^{1}$, desglosada en $1,28 \%$ para clorhidrato de cocaína (CC) y 0,62\% para pasta base de cocaína (PBC). La PBC es una sustancia intermedia en la producción de clorhidrato de cocaína, formada por sulfato de cocaína más distintos hidrocarburos ${ }^{2,3}$. En la región andina, la PBC presenta un consumo endémico en las últimas tres décadas ${ }^{2,4}$. Estudios nacionales de población general han mostrado que la PBC es la sustancia ilegal que genera mayor dependencia, alcanzando dos tercios de los consumidores del último mes ${ }^{5,6}$.

Sólo 7,2\% de los que abusan de drogas ilegales ingresan anualmente a tratamiento ${ }^{6}$, en parte porque ellos o su entorno no perciben el consumo como problemático o fuera de control ${ }^{6}$. Otros presentan, asociado al consumo, conductas socialmente sancionadas, alejándose de la posibilidad de contactar a los servicios sanitarios. Este grupo de sujetos marginados de los sistemas sociales de apoyo son denominados población oculta ${ }^{7}$.

La caracterización clínica de los consumidores de drogas ilegales es compleja, dado que los estudios de población general suelen subrepresentar a los sujetos con conductas socialmente sancionadas $^{7}$ y los estudios en población consultante caracterizan una proporción baja y seleccionada de ellos ${ }^{6}$. Respecto del consumo de CC y PBC en Chile, la caracterización clínica proviene de los estudios nacionales de $\operatorname{CONACE}^{1,5,6}$ y de unos pocos estudios de población clínica ${ }^{8-11}$ que no profundizan en patrones de consumo ni conductas de riesgo asociadas. Por tanto, el estudio de consumidores no consultantes es un complemento necesario para el mejor conocimiento de la clínica del consumo de CC y PBC, hasta el momento escasamente descrita.

Se han desarrollado diversas metodologías noprobabilísticas que permiten acceder y caracterizar a consumidores no consultantes ${ }^{7}$. El desafío de dichas metodologías es lograr representatividad a través de un riguroso control de sesgos ${ }^{12}$. La ¿Entrevista de Acceso Privilegiado»(EAP) ${ }^{13,14}$, una de dichas metodologías, recolecta información a través de entrevistadores que tienen acceso a los sujetos que se pretende estudiar. Santis y cols ${ }^{15,16}$, empleando la EAP en Chile, caracterizaron a consumidores crónicos de $\mathrm{PBC}$, no consultantes, describiendo su vulnerabilidad social y conductual.

La línea de investigación, de la cual es parte este reporte, tiene por objetivos conocer el perfil biopsicosocial de los consumidores de cocaína que no consultan espontáneamente e identificar prospectivamente los factores facilitadores y las barreras para el contacto con los servicios de rehabilitación. La hipótesis general de la investigación es que los consumidores de PBC presentan un mayor ocultamiento que los consumidores de CC a dichos servicios. Este artículo explora dos hipótesis: 1) El patrón de consumo de sustancias de los consumidores de PBC es de mayor gravedad que el de los consumidores de CC y, 2) Los consumidores de PBC presentan mayor frecuencia de conductas de riesgo que los consumidores de CC. Por tanto, los objetivos de este artículo son comparar el patrón de consumo y el perfil de conductas de riesgo en la evaluación inicial de estudio prospectivo de consumidores de PBC y de CC no consultantes a centros de rehabilitación.

\section{PACIENTES Y MÉTODO}

Diseño. Estudio prospectivo con tres evaluaciones: al reclutamiento, a los 6 y 12 meses de seguimiento. Se empleó la Entrevista de Acceso Privilegiado ${ }^{23,14}$ para el reclutamiento muestral. Esta investigación fue aprobada por el Comité de Ética de la Facultad de Medicina, Pontificia Universidad Católica de Chile.

Contexto. Se eligieron las 4 comunas de mayor consumo de PBC y CC en la Región Metropolitana de Chile, según el último estudio nacional con representatividad comunal ${ }^{5}$ : Pedro Aguirre Cerda, Huechuraba, La Pintana y San Joaquín. Se confeccionaron mapas de riesgo que delimitaron zonas territoriales de alto consumo y tráfico de drogas en cada comuna, con información de organizaciones locales y de CONACE.

Participantes. El muestreo fue estratificado por género (2/3 masculino), rango etáreo (menores de 19 años, entre 19 y 25, y mayores de 25; divididos en tercios) y dirigido a las zonas delimitadas por los mapas de riesgo (1/4 de sujetos en cada comuna). 
Criterios de inclusión: Grupo 1: al menos un consumo de $\mathrm{PBC}$ en el último mes; mayor frecuencia de consumo de PBC por sobre CC, en los últimos 30 días, en caso de coexistir. Grupo 2: al menos un consumo de CC en el último mes; mayor frecuencia de consumo de CC por sobre $\mathrm{PBC}$, en los últimos 30 días, en caso de coexistir. Ambos grupos sin tratamiento por consumo de sustancias en los últimos 6 meses.

El tamaño muestral fue calculado en base a variables continuas, con una potencia de $80 \%$ y significación de 5\%. La muestra incluyó 467 sujetos: 231 consumidores de PBC y 236 consumidores de CC.

Mediciones. La «Encuesta de Conductas de Riesgo» (ECR) fue construida por los investigadores en base al cuestionario Maudsley Addiction Profile ${ }^{17}$ y al utilizado en el Estudio Suizo de Poblaciones Ocultas $^{18}$. La ECR compila 246 ítems que evalúan: datos sociodemográficos; patrón de consumo de sustancias, dependencia de sustancias según criterios CIE-1019; conductas de riesgo; situación legal; percepción de servicios de salud. Se complementó con el cuestionario de salud general de Goldberg $\left(\right.$ GHQ-12) ${ }^{20}$; y el «Cuestionario Breve Cómo es SU/TU Familia»¹.
El análisis de confiabilidad test-retest de la ECR (coeficiente Kappa de Cohen), mostró que 85\% de los ítems tuvo un grado de acuerdo «Bueno»o «Muy Bueno»2, en los 40 ítems seleccionados, que son de respuesta general o preguntas de entrada a las distintas secciones. La consistencia interna de la ECR mostró en 11 de los 13 constructos un Alpha de Cronbach superior a 0,6, valor aceptable según González-Saiz ${ }^{23}$ (Tabla 1).

Los objetivos se operacionalizaron a través de las siguientes variables:

- Patrón de consumo: sustancias consumidas en el último mes y número de días de consumo en último mes; presencia de dependencia: 30 más de 6 criterios de la CIE- $10^{19}$ en los últimos 12 meses; severidad de dependencia: número de criterios de dependencia CIE-10 cumplidos; policonsumo: consumo de dos o más sustancias en el último mes; polidependencia: presencia de 2 o más dependencias (ambos excluye tabaco); número de dependencias y edad de inicio de consumo para cada sustancia.

- Conductas de riesgo: prevalencia de vida de sobredosis, autolesiones, intentos de suicidio, conductas sexuales, negligencia en el cuidado de menores y conducta antisocial.

Tabla 1. Índice de consistencia interna mediante el coeficiente Alpha de C ronbach para las distintas secciones del instrumento Encuesta de Conductas de Riesgo aplicado a 467 sujetos consumidores de sustancias ilícitas

\begin{tabular}{|lc|}
\hline Constructo & Alpha de Cronbach \\
\hline ¿Cómo es SU/TU familia? & 0,965 \\
Versión SU & 0,884 \\
Versión TU & 0,631 \\
Patrón de consumo de sustancias & 0,866 \\
Criterios de dependencia CIE-10 & \\
Conductas de riesgo & 0,562 \\
Sobredosis & 0,629 \\
Conducta sexual & 0,683 \\
Autolesiones & 0,476 \\
Intento de suicidio & 0,686 \\
Riesgo a terceros & 0,686 \\
Conducta laboral & 0,705 \\
Conducta antisocial & 0,757 \\
Situación legal & 0,734 \\
Salud y contacto con servicios de salud & \\
\hline
\end{tabular}


Procedimientos. A. Selección de los entrevistadores de acceso privilegiado. 1. Se convocó, a través de redes comunales formales, a personas que vivieran en las áreas de riesgo o tuvieran contacto con consumidores. 2. Preselección: 123 sujetos respondieron la valoración de habilidades sociales (Inventario de Comportamiento Interpersonal, ICI) ${ }^{24}$ y de comprensión lectora (Test de Cloze) ${ }^{25}$. 3. Entrevista semiestructurada: se evaluó a 64 sujetos su motivación para participar, características personales, percepción de riesgo y estrategias de afrontamiento ante situaciones de riesgo. 4. Fueron capacitadas 47 personas sobre la estructura y administración del instrumento. Finalmente, se seleccionó 28 entrevistadores: 18 mujeres y 10 hombres. El análisis de las características sociodemográficas de los entrevistadores sobre la selección muestral no reveló diferencias significativas relevantes entre los grupos.

B. Reclutamiento de los consumidores. Los entrevistadores contactaron 609 sujetos en las 4 comunas. De ellos, 122 declinaron participar; éstos contestaron una encuesta con información sociodemográfica básica y patrón de consumo de sustancias en último mes. El 83,6\% de estos rechazos cumplían los criterios de inclusión.

Posterior al consentimiento informado de cada sujeto, se administró la ECR en forma anónima, en diferentes lugares (calle, casa, plazas), y grabada en cinta de audio que fue revisada para comprobar la concordancia con los datos registrados. Se obtuvo 487 encuestas (tasa de respuesta 80\%), de las cuales se descartaron 20 por discordancia con la grabación. Se pagó $\$ 6.000$ por cada encuesta correctamente administrada; los sujetos evaluados no recibieron pago.

El 10\% de sujetos fue reentrevistado, una semana después, por un entrevistador gold standard. En los ítems categóricos, hubo acuerdo sustancial o cuasi perfecto del coeficiente Kappa de Cohen en 45,9\% y en $30 \%$ fue moderado 22 . Las variables continuas presentaron en $70,2 \%$ una correlación significativa (coeficiente de Spearman).

\section{Resultados}

Características sociodemográficas. Ambos grupos de consumidores eran predominantemente solte- ros y casi $70 \%$ vivía con su familia de origen. Los consumidores de PBC presentaron menor instrucción, mayor deserción escolar, mayor cesantía y empleo informal que los consumidores de CC (Tabla 2).

Patrón de consumo. El policonsumo fue mayoritario en ambos grupos, siendo la marihuana y el alcohol las sustancias coexistentes más frecuentes. El grupo 2 consumía alcohol y anfetaminas con mayor frecuencia que el grupo 1.

En todos los casos, la vía de administración para la PBC y marihuana fue la pulmonar y para CC la vía nasal.

Ambos grupos eran en alta proporción dependientes de las sustancias consumidas en el último mes y predominantemente polidependientes. Los sujetos del grupo 1 fueron dependientes de PBC en mayor proporción que los consumidores de PBC del grupo 2 (Tabla 3).

La severidad de dependencia de PBC del grupo 1 era mayor que la severidad de la dependencia de CC en el grupo 2 (5,5 versus 5,1: t-test: $\mathrm{t}(439)=4,7, \mathrm{p}<0,001)$. La severidad de la dependencia de los dependientes de PBC del grupo 1 fue mayor que la de los dependientes de PBC del grupo 2, y, la de los dependientes de CC del grupo 2 fue mayor que la severidad de los dependientes de CC del grupo 1 (Tabla 4).

Los sujetos del grupo 1 consumían casi diariamente PBC y más frecuentemente que los consumidores de PBC del grupo 2. El consumo de CC en el grupo 2 alcanzó una frecuencia de un tercio del mes, duplicando el consumo de usuarios de CC del grupo 1. Ambos grupos consumían marihuana dos tercios de los días del mes. La primera sustancia consumida en ambos grupos fue la marihuana, pero el grupo 1 se inició en el consumo de todas las sustancias ilícitas exploradas a edad más temprana (Tabla 4).

Conductas de riesgo. El grupo 1 reportó una mayor proporción que el grupo 2 en las siguientes conductas de riesgo en la vida (Tabla 5): sexuales (nunca uso de anticoncepción, sexo por dinero y droga por sexo), autolesiones, intento de suicidio, negligencia en el cuidado de menores, comisión de delitos y porte de arma. El perfil obtenido mediante regresión logística, mostró que cometer autolesiones (Odds Ratio (OR) $=1,87$; 95\% IC: 
Tabla 2. Variables sociodemográficas de consumidores de pasta base de cocaína (grupo $1=231$ sujetos) y consumidores de clorhidrato de cocaína (grupo 2 =236 sujetos)

\begin{tabular}{|c|c|c|c|c|}
\hline & \multicolumn{2}{|c|}{ Grupo 1} & \multicolumn{2}{|c|}{ Grupo 2} \\
\hline & $\mathrm{N}$ & $\%$ & $\mathrm{~N}$ & $\%$ \\
\hline \multicolumn{5}{|l|}{ Estado civil } \\
\hline Soltero & 188 & 81,4 & 178 & 75,4 \\
\hline Casado & 7 & 3,0 & 16 & 6,8 \\
\hline Separado/anulado & 10 & 4,3 & 15 & 6,4 \\
\hline Conviviente & 26 & 11,3 & 27 & 11,4 \\
\hline \multicolumn{5}{|l|}{ Vive con } \\
\hline Familia origen & 161 & 69,7 & 164 & 69,5 \\
\hline Familia nuclear & 20 & 8,7 & 29 & 12,3 \\
\hline Sólo pareja & 9 & 3,9 & 8 & 3,4 \\
\hline Otro & 24 & 10,4 & 26 & 11,0 \\
\hline Sólo & 7 & 3,0 & 9 & 3,8 \\
\hline Sin domicilio fijo & 10 & $4,3^{*}$ & 0 & $0,0^{*}$ \\
\hline \multicolumn{5}{|l|}{ Nivel educacional } \\
\hline Sin educación formal & 5 & $2,2^{*}$ & 0 & $0,0^{*}$ \\
\hline Básica completa/incompleta & 116 & $50,2^{*}$ & 48 & $20,3 *$ \\
\hline Media completa/incompleta & 106 & $45,9 *$ & 138 & $58,5^{*}$ \\
\hline Técnicos o universitarios & 4 & $1,7 *$ & 50 & $21,2^{*}$ \\
\hline Deserción escolar & 172 & $74,5^{\text {*⿻* }}$ & 92 & $39,0^{* * *}$ \\
\hline \multicolumn{5}{|l|}{ Situación Laboral } \\
\hline Cesante & 107 & $46,3^{*}$ & 64 & $27,1^{*}$ \\
\hline Trabajo informal & 75 & $32,5^{*}$ & 45 & $19,1^{*}$ \\
\hline Trabajo formal & 26 & $11,3^{*}$ & 84 & $35,6^{*}$ \\
\hline Estudiante & 23 & $10,0^{*}$ & 43 & $18,2^{*}$ \\
\hline
\end{tabular}

$* \mathrm{Chi}^{2}$ (residuos estandarizados), $\mathrm{P}<0,01$. ${ }^{* * *} \mathrm{Chi}^{2}, \mathrm{P}<0,001$.

1,18-2,94) y delitos (OR =2,07; 95\% IC: 1,36-3,17) se asocia a consumo de PBC más que a consumo de CC.

El 13,1\% de la muestra comunicó problemas actuales con la justicia, siendo mayor la proporción en el grupo $1(18,6 \%)$ que en el grupo 2 $(7,6 \%)\left(\mathrm{Chi}^{2}(1, \mathrm{n}=467)=12,4 ; \mathrm{p}<0,001\right)$.

\section{DISCUSIÓN}

Los especialistas en adicciones observan frecuentemente la mayor severidad del consumo de PBC que del consumo de CC. Nuestros datos avalan tal observación, que contaba con escasa evidencia empírica nacional. Además, comprueban la eficacia de la EAP para contactar consumidores en su ambiente natural. Las características del patrón de consumo y de las conductas de riesgo de consumidores no consultantes son clínicamente relevantes, dado que son éstos los potenciales usuarios de la red nacional de servicios de rehabilitación. En ese sentido, destacamos la alta frecuencia de consumo en ambos grupos, lo que determina, en la práctica, que estos sujetos estén bajo los efectos de las drogas o recuperándose de ellas de manera casi permanente. El mayor consumo compulsivo de $\mathrm{PBC}$, probablemente, se relaciona a la vía de administración pulmonar de esta droga, que determina mayores y más rápidos niveles plasmáticos de cocaína en comparación a la vía nasal del $\mathrm{CC}^{26,27}$. En los consumidores de PBC observamos mayor prevalencia de vida de la mayoría de las conductas de riesgo estudiadas, destacando las 
Tabla 3. Patrón de consumo de sustancias en el último mes de consumidores de pasta base de cocaína (grupo 1 =231 sujetos) y consumidores de clorhidrato de cocaína (grupo 2 =236 sujetos)

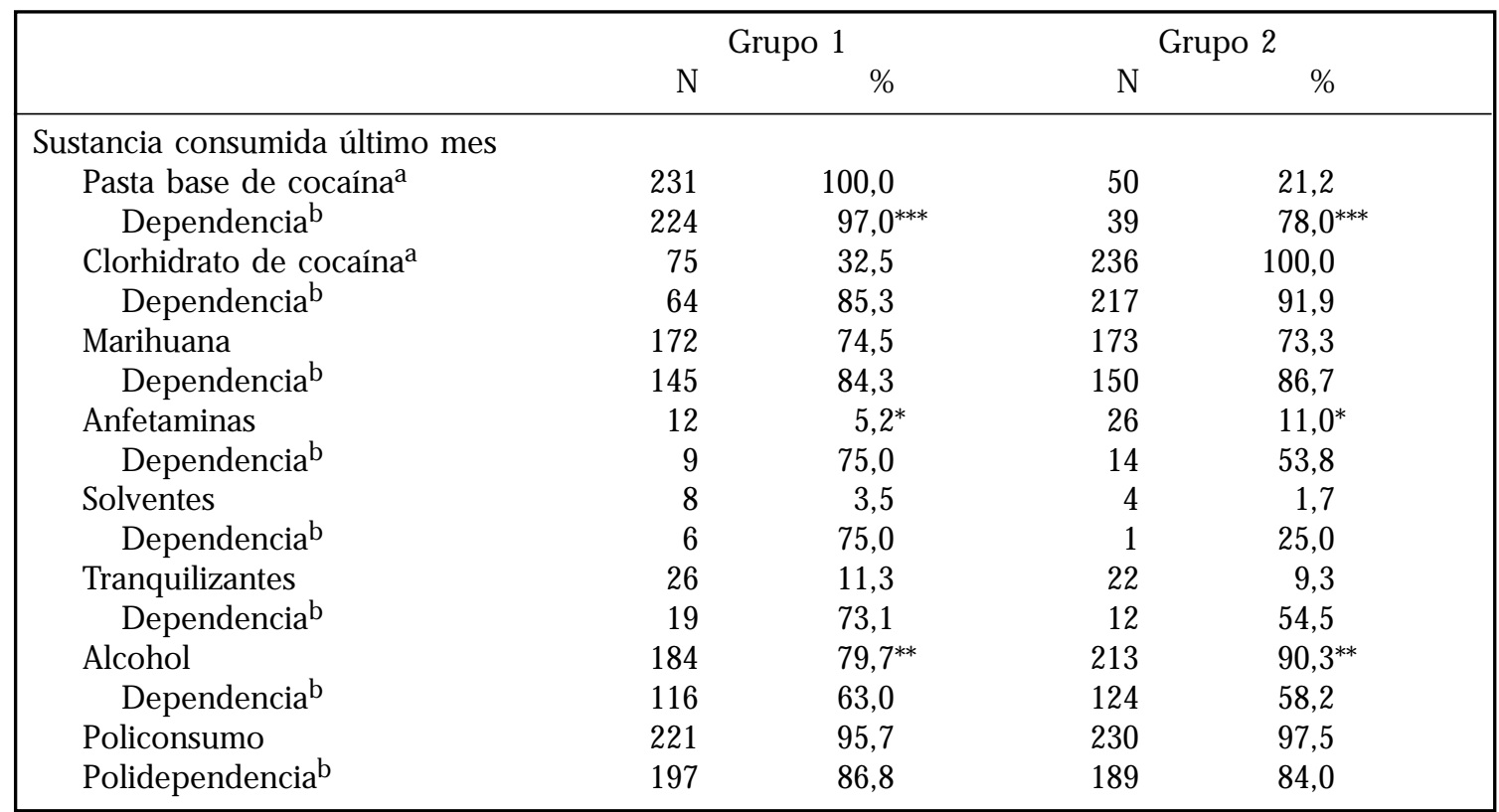

avariables de inclusión muestral. be acuerdo a criterios CIE-10 ${ }^{15}$, aplicados a consumidores de último mes. ${ }^{\dagger} \mathrm{Chi}^{2}$ (residuos estandarizados), $\mathrm{P}<0,01$.

${ }^{*} \mathrm{Chi}^{2}, \mathrm{P}<0,05 .{ }^{*} \mathrm{Chi}^{2}, \mathrm{P}<0,005 .{ }^{*} * \mathrm{Chi}^{2}, \mathrm{P}<0,001$.

autolesiones, intentos de suicidio y conductas antisociales. Estas conductas debieran ser evaluadas sistemáticamente, en especial en los consumidores de $\mathrm{PBC}$, por el riesgo vital personal y de terceros que implican.

Nuestros resultados son razonablemente extrapolables a los sujetos consumidores de PBC y CC de las áreas comunales consideradas, dada la validez y confiabilidad de la información recogida.

Destacamos que nuestros datos muestran diferencias importantes con el perfil de los consumidores en tratamiento descrito por CONACE (CONACE. Seguimiento de una cohorte de beneficiarios para la evaluación de impacto de los planes de atención de las instituciones en convenio con CONACE-FONASA. 2005; observaciones no publicadas). Los consumidores de PBC no consultantes aparecen con menores niveles de escolaridad, mayores niveles de desempleo, inicio más precoz del consumo de sustancias, mayor proporción de policonsumo y menos problemas con la justicia a pesar de la alta proporción de sujetos que delinquen. Sin embargo, los consumidores de CC presentan características similares a los sujetos en tratamiento.

Las características biopsicosociales descritas representan sólo asociaciones que no nos permiten inferir causalidad. Los factores que podrían determinar mayor gravedad de los consumidores de PBC sobre los de CC pueden ser múltiples. La vía de administración pulmonar de PBC, ha sido relacionada con mayor severidad de la adicción ${ }^{26,27}$. La PBC contiene sustancias tóxicas derivadas de hidrocarburos ${ }^{4}$, que pudieran tener algún rol en la severidad de la adicción. Otro factor es el peor funcionamiento familiar observado en los consumidores de PBC que en los consumidores de CC (Hidalgo C.G., Santis R, Rodríguez J, Hayden V, Anselmo E. Family functioning of out-of-treatment cocaine base paste and cocaine hydrochloride Users. Addictive Behavior 2006. Sometido a publicación). Es conocido que crecer en familias poco protectoras puede potenciar el riesgo de consumo de sustancias $^{28}$ y de dependencia ${ }^{29}$. La mayor 
Tabla 4. Variables continuas del patrón de consumo de sustancias ilícitas de consumidores de pasta base de cocaína (grupo 1 =231 sujetos) y consumidores de clorhidrato de cocaína (Grupo 2 =236 sujetos)

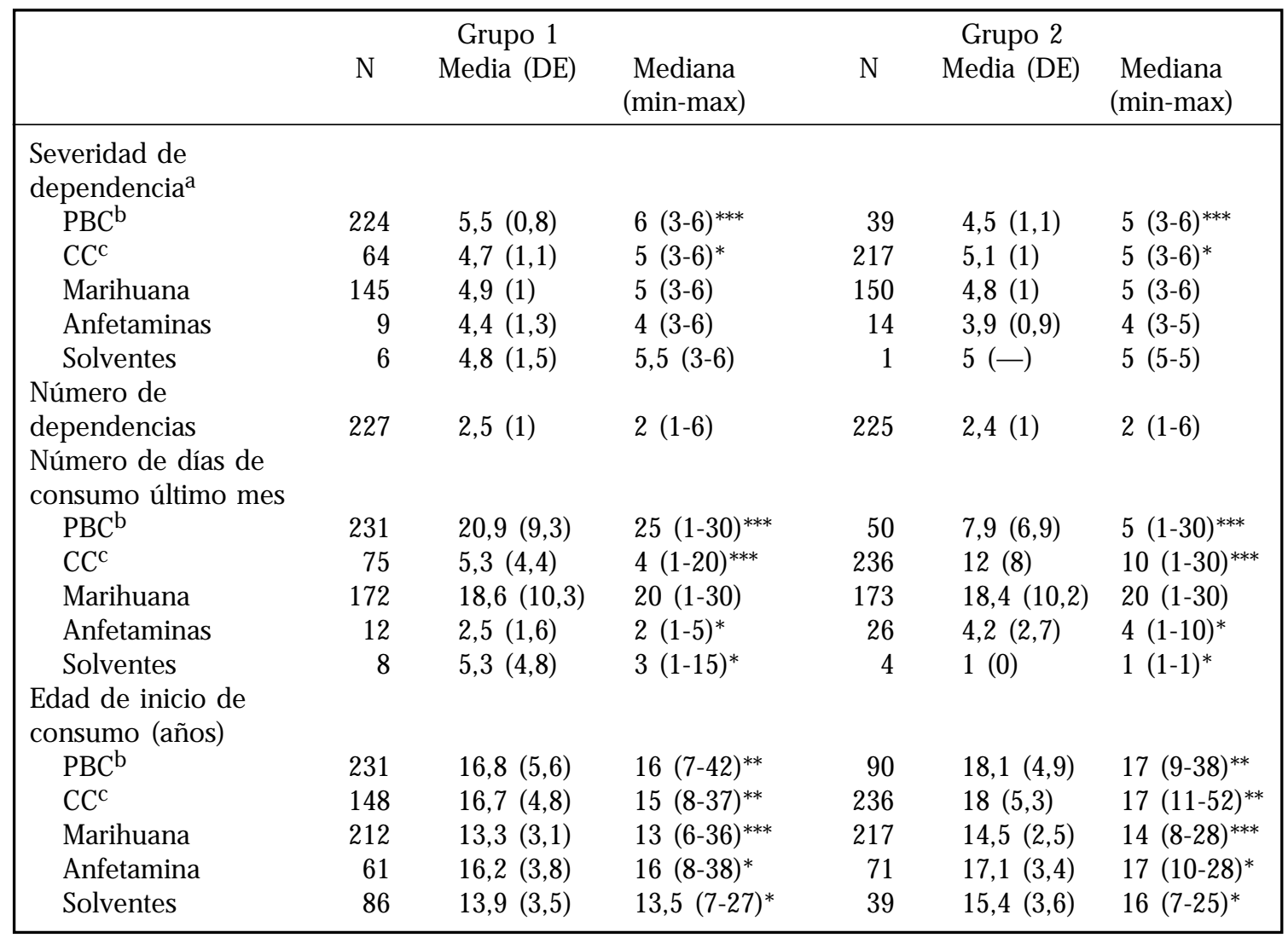

aDefinida como el número de criterios de dependencia cumplidos de acuerdo a la CIE-1015. bPasta base

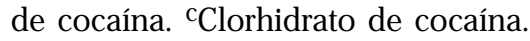

*Test de Mann-Whitney, $\mathrm{P}<0,05$. **Test de Mann-Whitney, $\mathrm{P}<0,005$. ***Test de Mann-Whitney, $\mathrm{P}<0,001$.

deserción escolar aumenta 5 veces la probabilidad de consumir drogas ${ }^{6}$. La peor situación laboral de los consumidores de PBC pudiera determinar un mayor riesgo para el consumo ${ }^{1}$. El diseño prospectivo de la investigación en curso podrá orientar respecto de la causalidad en las asociaciones encontradas.

Identificamos algunas limitaciones de nuestros resultados. La ECR está en proceso de perfeccionamiento, por lo que la validez de ciertos constructos es aún baja, específicamente intentos de suicidio y sobredosis. La valoración de dependencia debiera ser a través de entrevista clínica pero la descartamos por costo y riesgo de afectar la retención de sujetos. Tampoco, y por las mismas razones, corroboramos el consumo mediante análisis de drogas en fluidos corporales. Estas limitaciones plantean nuevos desafíos para el perfeccionamiento de la metodología.

Los resultados descritos están en la dirección de la hipótesis principal de nuestra línea de investigación en términos de que un porcentaje probablemente mayor de consumidores de PBC que de CC experimente, debido a su mayor marginalización y conductas de riesgo socialmente sancionadas, barreras que disminuyan la probabilidad de contacto con los servicios de rehabilitación al año de seguimiento. 
Tabla 5. Prevalencia de vida de conductas de riesgo de consumidores de pasta base de cocaína (grupo 1 =231 sujetos) y consumidores de clorhidrato de cocaína (grupo $2=236$ sujetos)

\begin{tabular}{|lrrrr|}
\hline & \multicolumn{2}{c}{ Grupo 1} & \multicolumn{2}{c|}{ Grupo 2} \\
Conductas de riesgo & $\mathrm{N}$ & $\%$ & $\mathrm{~N}$ & $\%$ \\
\hline Sobredosis drogas y/ o alcohol & 108 & 46,8 & 102 & 43,2 \\
Sexualmente activo & 217 & 93,9 & 224 & 94,9 \\
$\quad$ Nunca uso anticoncepción & 115 & $53^{* * *}$ & 75 & $33,5^{* * *}$ \\
Nunca uso preservativo & 124 & 57,1 & 102 & 45,5 \\
$\quad$ Riesgo contagio VIH & 108 & 49,8 & 109 & 48,7 \\
Droga por sexo & 36 & $16,6^{* *}$ & 17 & $7,6^{* * *}$ \\
$\quad$ Sexo por dinero & 31 & $14,3^{* *}$ & 14 & $6,3^{* *}$ \\
Autolesiones & 83 & $35,9^{* * *}$ & 46 & $19,5^{* * *}$ \\
Intento suicida & 75 & $32,5^{* * *}$ & 45 & $19,1^{* * *}$ \\
Cuidado actual de menores & 86 & 37,2 & 95 & 40,3 \\
$\quad$ Negligencia por consumo & 29 & $33,7^{* *}$ & 14 & $14,7 * *$ \\
Conductas antisociales & & & & \\
$\quad$ Porte arma fuego o cortante & 143 & $61,9^{* * *}$ & 109 & $46,4^{* * *}$ \\
Agresión a terceros con arma & 50 & 35 & 28 & 25,7 \\
Comisión delitos & 172 & $74,5^{* * *}$ & 132 & $55,9^{* * *}$ \\
\hline
\end{tabular}

${ }^{*}{ }^{*} \mathrm{Chi}^{2}, \mathrm{P}<0,005 .{ }^{* * *} \mathrm{Chi}^{2}, \mathrm{P}<0,001$.

La metodología empleada permitió conocer a este subgrupo de sujetos de alta vulnerabilidad en su ambiente natural, abriendo posibilidades para el

\section{REFERENCIAS}

1. CONACE. Sexto Estudio Nacional de Drogas en Población General de Chile. Ministerio del Interior, Gobierno de Chile 2004. Disponible en: http://www.conacedrogas.cl/docs obs. [Consultado el 27 de febrero de 2006].

2. JERI FR. Coca-paste smoking in some Latin American countries: a severe and unabated form of addiction. Bull Narc 1984; 36: 15-31.

3. El Sohly M, BrenNeisen R, Jones AB. Coca paste: Chemical analysis and smoking experiments. J Forensic Sci 1991; 36: 93-103.

4. Montoya I, Chilcoat H. Epidemiology of coca derivatives use in the Andean region: a tale of five countries. Subst Use Misuse 1996; 31: 1227-40.

5. CONACE. Cuarto Estudio Nacional de Drogas en Población General de Chile. Ministerio del Inte- desarrollo de intervenciones comunitarias destinadas a modificar el consumo de sustancias y las conductas de riesgo de consumidores no consultantes.

rior, Gobierno de Chile 2000. Disponible en: http://www.conacedrogas.cl/docs_obs. [Consultado el 27 de febrero de 2006].

6. CONACE. Quinto Estudio Nacional de Drogas en Población General de Chile. Ministerio del Interior, Gobierno de Chile 2002. Disponible en: http://www.conacedrogas.cl/docs obs. [Consultado el 27 de febrero de 2006].

7. WIEBEL $W$. Identifying and gaining access to hidden populations. NIDA Res Monogr 1990; 98: 4-11.

8. Hernández G, Montino O, Kimelman M, Oreliana G, NuÑEZ C, IBaÑEZ C. Prevalencia de trastornos psiquiátricos por uso de alcohol y otras sustancias en hombres y mujeres hospitalizados en medicina interna de un hospital de Santiago de Chile. Rev Méd Chile 2002; 130: 651-60.

9. Mena M, Navarrete P, Corvalán S, Bedregal P. Drogadicción embriofetal por abuso de pasta 
base de cocaína durante el embarazo. Rev Méd Chile 2000; 128: 1093-100.

10. Mena M, Corvalán S, Bedregal P. Gastos en salud de hijos de consumidoras de pasta base de cocaína. Rev Méd Chile 2002; 130: 1241-8.

11. PÉrez J. Clínica de la adicción a pasta base de cocaína. Rev Chil Neuro-psiquiatr 2003; 41: 55-63.

12. Van De Goor LA, Garretsen HF, Kapian C, Korf D, SPRUIT IP, De ZwaRT WM. Research methods for illegal drug use in hidden populations: summary report of a European invited expert meeting. J Psychoactive Drugs 1994; 26: 33-40.

13. Griffiths P, Gossop M, Powis B, Strang J. Reaching hidden populations of drug users by privileged access interviewers: methodological and practical issues. Addiction 1993; 88: 1617-26.

14. Santis R, Hayden V, Ruiz S, Anselmo E, Torres R, Pérez De Los Cobos J. Rendimiento de la entrevista de acceso privilegiado para caracterizar usuarios de pasta base de cocaína. Rev Chil NeuroPsiquiat 2004; 42: 273-80.

15. Santis R, Hayden V, Ruiz S, Anselmo E, Anuch C, BRonstein E et aL. Privileged access interviewing to characterize cocaine base paste users. Drug Alcohol Depend 2002; 66: S155.

16. Santis R, Hayden V, Ruiz S, Anselmo E, Torres R, HIDALGo CG. Patrones de consumo de sustancias de una muestra no consultante de consumidores de pasta base de cocaína. Rev Chil Neuro-psiquiat 2006; 44: 15-22.

17. Marsden J, Gossop M, Stewart D, Best D, Farrell M, Lehmann P Et al. The Maudsley Addiction Profile (MAP): a brief instrument for assessing treatment outcome. Addiction 1998; 93: 1857-67.

18. Kuebier D, Hausser D. The Swiss hidden Population Study: practical and methodological aspects of data collection by privileged access interviewers. Addiction 1997; 92: 325-334.

19. World Health Organization. The ICD-10 classification of mental and behavioural disorders: cli- nical descriptions and diagnostic guidelines. Geneva: WHO; 1992.

20. ARAYA R, Wynn R, LEwis G. Comparison of two self administered psychiatric questionnaires (GHQ-12 and SRQ-20) in primary care in Chile. Soc Psychiatry Psychiatr Epidemiol 1992; 27: 168-73.

21. Vaidés M, Rodríguez J, Serrano T. Adaptación de la escala abreviada del instrumento «Cómo es Tu familia-Breve» y «Cómo es Su familia-Breve» En O.P.S: Familia y adolescencia: indicadores de salud. Manual de aplicación del instrumento. Abreviado. Organización Panamenicana de la Salud. 1999.

22. LaNdis JR, Koch GG. The measurement of observer agreement for categorical data. Biometrics 1977; 33: 159-74.

23. González Saiz F, Iraurgi I, Caruma S. Teoría de la medida e instrumentos de evaluación en drogodependencia. Cuestiones Básicas. En: Instrumentos de Evaluación en Drogodependencias. Iraurgi I, González Saiz F. (Eds). Madrid. Aula Médica Ediciones. 2002.

24. Mauger P, Adkinson D. Interpersonal Behavior Survey (IBS). Manual. California. Western Psychological Service. 1987.

25. Condemarín M, Miucic N. Test de Cloze: Procedimiento Para el Desarrollo y la Evaluación de la Comprensión Lectora. Santiago. Editorial Andrés Bello. 1994.

26. Jones R. The Pharmacology of Cocaine Smoking in Humans. NIDA Res Monogr 1990; 99: 30-41.

27. Strang J, Bearn J, Farrell M, Finch E, Gossop M, GRIFFTHS P ET AL. Route of drug use and its implications for drug effect, risk of dependence and health consequences. Drug Alcohol Rev 1998; 17: 197-211.

28. Merikangas KR, Dierker L, Fenton B. Familial Factors and Substance Abuse: Implications for Prevention. NIDA Res Monogr 1998; 177: 12-41.

29. Cirilo S, Berrini R, Cambiaso G, Mazza R. Una Mirada a la literatura. En: Cirillo S, Berrini R, Cambiaso G, Mazza R, ed. La familia del toxicodependiente. Barcelona: Editorial Paidós, 1999; 21-45.

\section{Agradecimientos}

Agradecemos a FONDECYT por el financiamiento del proyecto Estudio prospectivo del impacto biopsicosocial del uso de pasta base de cocaína y clomidrato de cocaína en sujetos no consultantes a servicio de rehabilitación», objeto de este reporte (Proyecto FONDECYT 1030431). Agradecemos a los entrevistadores cuyo esfuerzo hizo posible este estudio. 